\title{
Soft Computing Applications in Dynamic Model Identification of Polymer Extrusion Process
}

\author{
Leong Ping Tan, Ahmad Lotfi, Eugene Lai and J.B. Hull \\ School of Engineering \\ Nottingham Trent University \\ Burton Street, Nottingham, NG1 4BU, United Kingdom \\ email:\{leong.tan, ahmad.lotfi, eugene.lai, barry.hull\}@ntu.ac.uk
}

\begin{abstract}
This paper proposes the application of soft computing to deal with the constraints in conventional modelling techniques of the dynamic extrusion process. The proposed technique increases the efficiency in utilising the available information during the model identification. The resultant model can be classified as a 'grey-box model' or has been termed as a 'semi-physical model' in the context. The extrusion process contains a number of parameters that are sensitive to the operating environment. Fuzzy ruled-based system is introduced into the analytical model of the extrusion by means of submodels to approximate those operational-sensitive parameters. In drawing the optimal structure for the sub-models, a hybrid algorithm of genetic algorithm with fuzzy system (GA-Fuzzy) has been implemented. The sub-models obtained show advantages such as linguistic interpretability, simpler rule-base and less membership functions. The developed model is adaptive with its learning ability through the steepest decent error back-propagation algorithm. This ability might help to minimise the deviation of the model prediction when the operational-sensitive parameters adapt to the changing operating environment in the real situation. The model is first evaluated through simulations on the consistency of model prediction to the theoretical analysis. Then, the effectiveness of adaptive submodels in approximating the operational-sensitive parameters during the operation is further investigated.
\end{abstract}

Keywords: Dynamic modelling, soft computing, GA-fuzzy algorithm, steepest decent error backpropagation, semi-physical modelling, distributed parameter system, adaptive, polymer extrusion. 


\section{Introduction}

Extrusion is a critical operation in the thermoplastic processing industry since all commercial polymers are compounded [1]. During the process of extrusion, the quality of the product fluctuates within a certain range due to the variation in the rheological properties of materials, or the operating environment including the system disturbances. This may introduce defects to the end product. A number of control methodologies have been proposed to regulate the product quality [2], [3], [4], [5]. A simple methodology can be a Single-Input-Single-Output (SISO) feedback control linking the output at the die to the input parameter such as the screw speed. However, this technique is not efficient as the product quality is greatly affected by the flow history inside the extruder. Further complication includes the strong interactions between process parameters. For example, changes in the screw speed can lead to changes not only in the melt temperature, but in the flow rate as well. Consequently, a feasible control methodology shall consider the process as a multivariable and distributed parameter system.

The design of an appropriate controller requires a dynamic model of the extrusion. The model development is restricted by the lack of access of some process parameters during the model identification. Some parameters inside the extruder, such as the true temperature of the melt are expensive, if possible, to be gauged directly due to the operational constraints. In common with many other chemical processes, the polymer processing introduces a few parameters that are improper to be expressed in a deterministic manner. These parameters are referred to as the 'operational-sensitive parameters'. For instance, the polymer viscosity can be regarded as the operational-sensitive parameter. The value of the parameter varies due to the inconsistency of the properties of the input material.

The aforementioned modelling problems limit the effectiveness of applying either empirical or analytical modelling technique in identifying the dynamic extrusion process. If the combination of analytical and empirical modelling techniques is possible, the flexibility will improve the utilisation of the available information rather than having the need to conduct a series of dedicated experiments for the model identification. It is speculated that the semi-physical or semi-mechanistic modelling technique can be a potential solution [6]. The technique shall integrate different sources of knowledge and technologies during the model development. 
In this paper, the Fuzzy Rule-Based System (FRBS) is applied as the sub-models to approximate the operational-sensitive parameters. The optimal structures of the sub-models are obtained by executing the GA-Fuzzy algorithm. The sub-models thus obtained are integrated with the analytical model, forming a semi-physical model. The semi-physical model is adaptive to the varying operating environment through its learning ability while preserving the multivariable and distributed nature of the process. In the following sections, a brief description of the extrusion process will be given, to be followed by soft computing applications in developing a semi-physical model. The model evaluation is performed from two bases. Firstly, the predictions on the melt temperature, pressure and mass-flow rate are compared to the theoretical analysis. Then, the effectiveness of adaptive sub-models in approximating the operational-sensitive parameters is further investigated.

\section{The Process Description}

The schematic diagram of a single screw extruder is illustrated in Figure 1. The extruder consists of a barrel, which is heated by a series of wall-mounted heaters and a rotating screw; the latter is linked to a gear system and a motor. Polymer particles are fed in through the hopper, by gravity, into the helical screw channel. The screw has a varying channel depth along the axial direction. The melt flowing through the die is formed into an end product. Figure 2 illustrates the content of a typical helical cross section of the screw channel in the compression section. It consists of a solid bed and a melt pool. The size of the solid bed decreases as it travels in the axial direction and eventually diminishes when it reaches the metering section. The variation of the width of the solid bed along the screw channel in helical direction is called the Solid Bed Profile (SBP) while the variation of the temperature of the melt along the helical screw channel is called the Melt Temperature Profile (MTP).

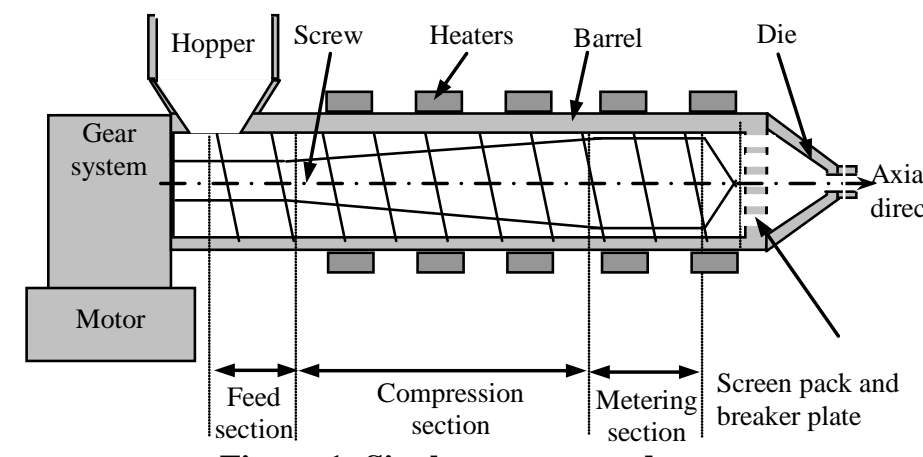

Figure 1: Single screw extruder

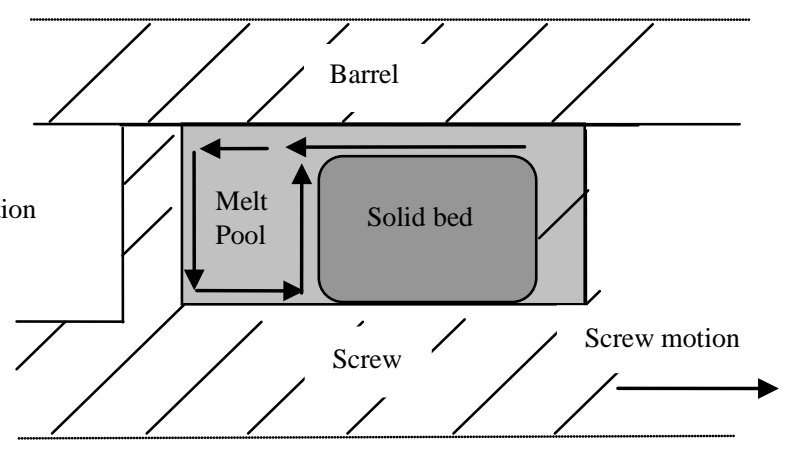

Figure 2: Melting of solid bed 


\section{The Development of Semi-physical Dynamic Extrusion Model}

An analytical dynamic model of the extrusion process is available [7]. However, its application in the controller design is limited since it is not adaptive to the varying operating conditions. The variation could be due to the instability of the power supply affecting the screw speed and barrel temperature, the changes of ambient conditions such as humidity, temperature, etc. In addition, the properties of the polymer to be processed may vary from batch to batch, depending on the regrind level, drying condition, etc. A number of process parameters are sensitive to the variation, particularly the polymer rheology during the extrusion. The rheology is approximated by means of a power law model in the analytical model. The parameters of the power law model are predetermined based on the experiments conducted on a rheometer. The application of the power law model to describe the polymer rheology has several shortcomings. Its validity is limited to the intermediate shear rate range and its parameters are not adaptive accordingly to the variation of the operating conditions. In the present study, a semiphysical model is developed by extending the analytical model so that the FRBS is applied as submodels to approximate the operational-sensitive parameters.

Figure 3 provides an overview of development of the semi-physical model for the extrusion process [8]. The analytical model provides simulated data only for the initialisations of the FRBS sub-models. The optimal sub-models thus obtained are incorporated into the analytical model forming the semi-physical model. The model is then simulated and the predictions are compared to the experimental measurements on the melt temperature and the melt pressure. If there is any error exists, a model adaptation algorithm will commence to minimise the error by tuning the parameters of the FRBS sub-models.

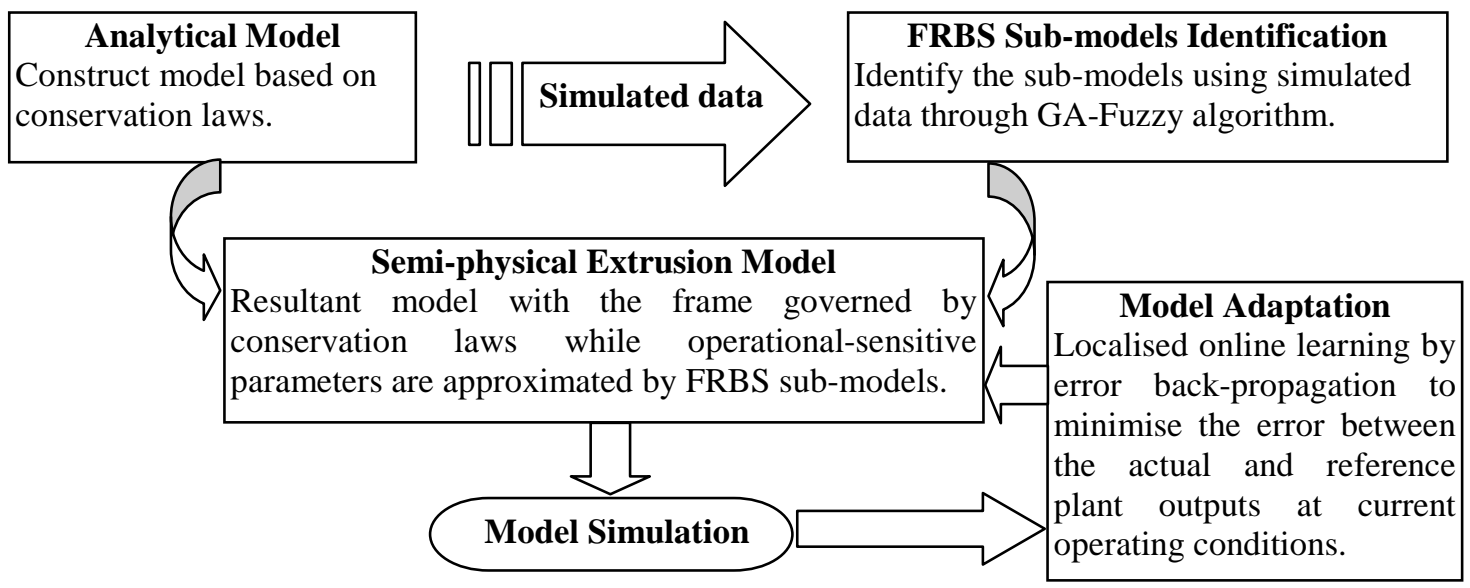

Figure 3: Overview of development of the semi-physical model. 


\subsection{The analytical model}

The semi-physical dynamic model in the case study is an extension of the analytical model of the work in [7]. The following assumptions are made for the analytical model:

- The states of the process dynamic can be described by the transient responses of the Solid Bed Profile (SBP), represented as $X(t, z)$ and the Melt Temperature Profile (MTP), represented as $T(t, z)$ where $z$ and $t$ are the helical down channel distance and time instant respectively. Dynamic condition prevails only when the melting starts, i.e. in the melting and melt conveying mechanisms;

- Local melting rate, heat transfer rate through barrel wall, viscous heat dissipation rate and pressure profile (PP) can be calculated using steady-state equations accordingly to local instantaneous conditions.

On the basis of the above assumptions, the analytical dynamic model is formulated. Two main equations in which the dynamic SBP governed by the differential mass balance on the solid bed is expressed in Equation 1 whereas the dynamic MTP governed by the differential energy balance on the melt pool is written as Equation 2.

$$
\begin{aligned}
& \frac{\partial X}{\partial t}+v_{\mathrm{sz}} \frac{\partial X}{\partial z}=-\frac{\phi X^{1 / 2}}{\rho_{s} H}+\frac{A v_{s z}}{H} X \\
& \frac{\partial T}{\partial t}+v_{m z} \frac{\partial T}{\partial z}=\frac{\phi X^{1 / 2}\left(T_{f}-T\right)}{\rho_{m} H(W-X)}+\frac{q_{t r}+q_{v c}}{C_{m} \rho_{m} H(W-X)}
\end{aligned}
$$

where $X$ is the solid bed width and $T$ is the melt temperature; $v_{s z}$ and $v_{m z}$ are the down channel velocity for the solid and melt respectively; $\rho_{s}$ and $\rho_{m}$ are the density of solid and melt; $A$ is the slope of compression section; $H$ is the channel height; $T_{f}$ is the film temperature; $\phi$ is the melting coefficient; $C_{m}$ is the heat capacity of the melt; $W$ is the channel width; $q_{t r}$ is the heat transfer rate through barrel wall; $q_{v c}$ is the viscous heat dissipation rate.

The parameters affected by the rheology in the model are $\phi$ and $q_{v c}$. Similar concern is also found for $q_{t r}$ due to the uncertainty in the heat transfer duration. These parameters have been expressed in the analytical model as the functions as shown in the following equations [9]: 


$$
\begin{aligned}
& \phi=f_{\phi}\left(\omega, T_{b}, G, \alpha\right) \\
& q_{t r}=f_{q_{t r}}\left(\omega, T_{b}, X, T, \alpha\right) \\
& q_{v c}=f_{q_{v c}}(\omega, T, X, G, \alpha)
\end{aligned}
$$

where $\omega$ is the screw speed, $T_{b}$ is the barrel temperature, $G$ is the total mass flow rate, $\alpha$ is the parameters specifying the physical properties of polymer and the geometry of the extruder. Within the parameter set of $\alpha$, the apparent viscosity $\eta$ is an important element that describes the physical properties of the molten polymer. The value of $\eta$ has been represented by the power law model with reference to the local melt temperature and the predetermined parameter set $\theta$. The equation can be simplified as:

$$
\eta=f_{\eta}(T, \theta)
$$

where $\theta$ is the parameter set predetermined by the offline experiments on a rheometer.

The deterministic equations (Equations 3 to 6) used in the analytical model to describe these varying operational-sensitive parameters are considered inappropriate, since they are not adaptive when the actual parameters are varying. The FRBS is introduced as an alternative to describe the behaviours of those parameters.

\subsection{The FRBS sub-models identification}

Equations 7 to 10 express the fuzzy functions of the operational-sensitive parameters. A hybrid GAFuzzy algorithm is adopted to identify the optimal structure for the FRBS sub-models. Data from the simulation of the analytical model is used as the training data during the identification process.

$$
\begin{aligned}
& \phi=f_{f z}\left(\omega, T_{b}\right) \\
& q_{t r}=f_{f z}\left(\omega, T_{b}, X\right) \\
& q_{v c}=f_{f z}\left(\omega, T_{b}, T, H\right) \\
& \eta=f_{f z}(\omega, X, T)
\end{aligned}
$$

where $f_{f z}$ is the fuzzy function. 


\section{The GA-Fuzzy algorithm}

The GA-Fuzzy algorithm adopted is similar to the Pittsburgh approach [10]. In the algorithm, each chromosome in the population represents a complete Knowledge Base (KB), which consists of Data Base (DB) and Rule Base (RB). The potential solution for a sub-model of $n$ input, $m$ output and $k$ rules is coded into a chromosome with a fixed binary number of $l$ bits. Each variable of the input and output has its own set of Membership Functions (MFs). The identification process begins with a sub-model of maximum MFs, i.e. $q_{i}=k, i=1 \ldots n+m$. As the FRBS sub-models are of zero-order Sugeno type with symmetric Gaussian MFs (two MF parameters), the total length of a chromosome is $L=l(2 \mathrm{x}(m+n) \mathrm{x}$ k). The algorithm is programmed with a flexible coding length to cope with the deducting number of rules and MFs during the structure optimisation. Apart from the chromosome representation, the genetic operators are important to enrich the possible solutions for the optimal structure. The commonly used operators include selection, crossover and mutation.

\section{The optimisation process}

Figure 4 shows the flow diagram for the optimisation of the structure of a FRBS sub-model. The structure is defined as a number of rules that constituting the sub-model and the way of each Membership Functions (MFs) of the input and output parameters associated in the rules. The initial setting defines the requirements of the optimisation. For example, the maximum number of rules desired, type of genetic operators, definition of convergences, etc. It is possible to translate the expert knowledge to the construction of the FRBS sub-models during the initial setting.

During the Data Base (DB) optimisation, fitness indices are assigned to all chromosomes within the population. The definition of the best fitness depends on a number of criteria as proposed by [11]. In the present study, the fitness index is determined by the inverse of mean square error between the predicted and desired result. A number of high fitness chromosomes are kept for the next generation and genetic operators are applied to the current population. The optimisation process is repeated until the convergent criterion is met. When this happens, the population with the selected chromosomes proceeds to Knowledge Base (KB) optimisation. 
The KB optimisation procedure is similar to that of the DB optimisation except that three extra fitness indices, namely the entropy of a FRBS $\left(F_{I}^{E}\right)$, the MF similarity $\left(F_{I}^{S}\right)$, and the rule activation $\left(F_{I}^{Z}\right)$, are included with predetermined weights and are evaluated in a single convergent criterion. Introducing $F_{I}^{E}$ into the criterion encourages an adequate overlapping of MFs of the sub-models, while $F_{I}^{S}$ and $F_{I}^{Z}$ search for similar MFs and the redundant rules of the sub-models.

Upon convergence of the KB optimisation, only the best chromosome is selected for the structure closure step. A new simpler structure is obtained by unifying similar Membership Functions (MFs) and deleting the redundant rules. Then, a new generation of the chromosomes having this new structure is created and the entire GA-fuzzy optimisation process is then repeated. While the convergences of Data Base (DB) and Knowledge Base (KB) optimisations maybe decided by the predefined number of generations or error limits, the optimisation of the structure of a sub-model is deemed to have converged only if the closure step cannot be further executed.

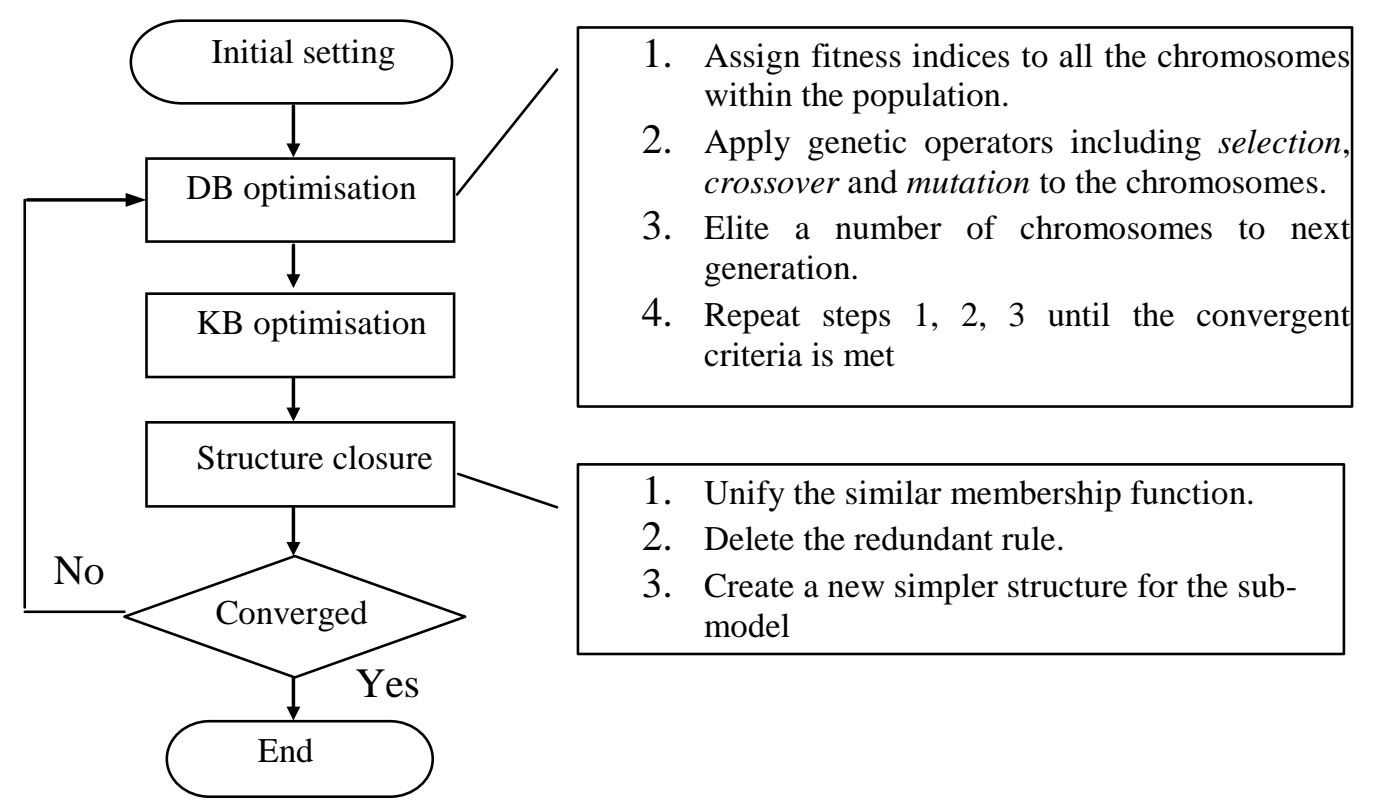

Figure 4: GA-Fuzzy algorithm for the optimisation of the structure of the sub-model 
Table 1: The setting of GA parameter and the resultant FRBS sub-models structure.

\begin{tabular}{|c|c|c|c|c|}
\hline Properties & $\phi$ sub-model & $q_{v c}$ sub-model & $q_{t r}$ sub-model & $\eta$ sub-model \\
\hline Number of inputs & 2 & 4 & 3 & 3 \\
\hline Size of population & 40 & 60 & 50 & 50 \\
\hline Variable bit number, $l$ & 6 & 6 & 6 & 6 \\
\hline Type of selection & Tournament & Tournament & Tournament & Tournament \\
\hline Type of crossover & Multiple-points & Multiple-points & Multiple-points & Multiple-points \\
\hline Crossover rate & 1 & 1 & 1 & 1 \\
\hline Mutation rate & 0.1 & 0.1 & 0.1 & 0.1 \\
\hline Elite chromosomes & 5 & 5 & 5 & 5 \\
\hline Initial number of rules & 27 & 36 & 30 & 30 \\
\hline Initial number of MFs & $27 \times 27 \times 27$ & $36 \times 36 \times 36 \times 36 \times 36$ & $30 \times 30 \times 30 \times 30$ & $30 \times 30 \times 30 \times 30$ \\
\hline Final number of rules & 5 & 3 & 5 & 2 \\
\hline Final number of MFs & $5 \times 5 \times 5$ & $2 \times 3 \times 2 \times 3 \times 3$ & $5 \times 5 \times 5 \times 5$ & $2 \times 2 \times 1 \times 2$ \\
\hline
\end{tabular}

Table 1 tabulates the setting of GA parameters during the structure identifications and the final structures obtained for the FRBS sub-models. The size of the population is set accordingly to the number of inputs in each sub-model. Larger population enhances the probability of variation, which is especially important for a lengthy chromosome. The parameters of MF are encoded into 6 bits binary code, in which a range of 64 discretised values is obtained. The resolution should be enough as the parameters represent only the width and the location of the MF within a bounded operating range. The strategy for applying the genetic operators is tournament selection, multiple-points crossover with the crossover rate of 1 and randomised mutation with a trigger weight function of 0.1. Elitism is applied as a special policy to each generation. The properties of elite chromosomes are exceptional and excluded from applying the genetic operators. 5 chromosomes of the highest fitness within the current generation are elite to the next generation. The policy might help to ensure the fitness of chromosomes in the next generation will be at least maintained same level, if not higher than, the current generation. To an extent, if all the chromosomes of the current generation are elite to the next generation, the fitness of the chromosomes of next generation will never be improved.

It is clear that the sub-models contain fewer MF and smaller number of rules. For example, the $q_{t r}$ submodel is initialised with 30 fuzzy rules and 30 MFs per variable. After the structure optimisation, the sub-model is reduced to 5 fuzzy rules and 5 MFs per variable. The advantages of the simpler structure include easier model interpretation, computation economical and efficiency in the model adaptation. 


\subsection{The model adaptation}

The sub-models with the optimised structures are incorporated into the semi-physical model for further adaptation. The commencement of the adaptation process is dependent on the errors of the model predictions when compared with the actual melt temperature and pressure. The model adaptation is complicated since the sub-models approximate only the operational-sensitive parameters but not the melt temperature and the pressure. These approximations will then be part of the semi-physical model in generating the predicted melt temperature and pressure as depicted in Figure 6. Therefore, the model adaptation is executed by back-propagating the prediction errors to the corresponding sub-model through level by level. The concept of error back-propagation from the prediction to each parameter of the Membership Function (MF) is shown in Figure 5. In the forward sequence (left to right), a small change in the MF parameter $c$ will affect the inferential membership grade of an input $w$. The influence is further reflected on the approximation of the FRBS sub-model $f_{f z}$, which will in turn affect the prediction of the semi-physical model, $\hat{o}$. It can be seen that the prediction error can be minimised if the effects of change at individual levels are known, which are the gradient vectors of the causal relationship. The derivative information is passed from the output level, and going backward level by level, to the first level as shown in the backward sequence (right to left) of Figure 5.

The total derivative information in the backward sequence can be effectively represented by a chain rule, which is generalised as Equation 11. MF parameters are then updated by the steepest descent algorithm. The tuning of the MF parameters is accomplished by minimising the sum of squared errors between the desired, $o$ and predicted, $o$ results as written in Equation 12.

$$
\begin{aligned}
& \frac{\partial J}{\partial c_{q i}^{p}}=\frac{\partial J}{\partial f_{f z}^{p}} \sum \frac{\partial f_{f z}^{p}}{\partial w_{k}^{p}} \times \frac{\partial w_{k}^{p}}{\partial c_{q i}^{p}} \\
& J=\Sigma(o-\hat{o})^{2}
\end{aligned}
$$

where $J$ and $p$ are the square error index and the sub-model indices respectively.

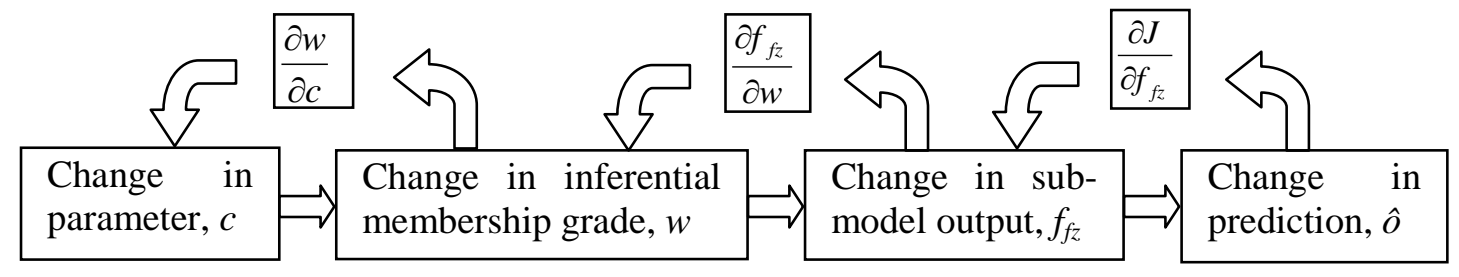

Figure 5: Causal relationship of MF parameters to the model prediction. 


\subsection{The model simulation}

Figure 6 illustrates the computation path of the semi-physical dynamic model. The program is initially provided with the following input data:

- Geometry parameters such as channel depth;

- Material properties such as density of solid polymer;

- Barrel temperature setting as a function of time, $T_{b}(t)$;

- Screw speed as a function of time, $\omega(t)$;

- Initial profiles for the solid bed and melt temperature, $X(z, t-1), T(z, t-1)$;

- The location where the melting process commences;

- Initial estimate of the output flow rate, $G(t)$.

The output flow rate, $G$ is initialised with the flow rate taken from the previous steady-state condition. When the operating condition changes with time, the program is iterated until a new steady-state condition is reached. The estimation on the output flow rate improves during the iteration. The relationship between flow rate and pressure-drop at the die, $\Delta P_{\text {die }}$ is shown in Equation 13.

$$
G=K_{\text {die }} \rho_{m} \frac{\Delta P_{\text {die }}}{\eta_{\text {die }}}
$$

where $K_{\text {die }}$ and $\rho_{m}$ are the die constant and density of melt respectively while $\eta_{\text {die }}$ is the apparent viscosity of the melt at the die.

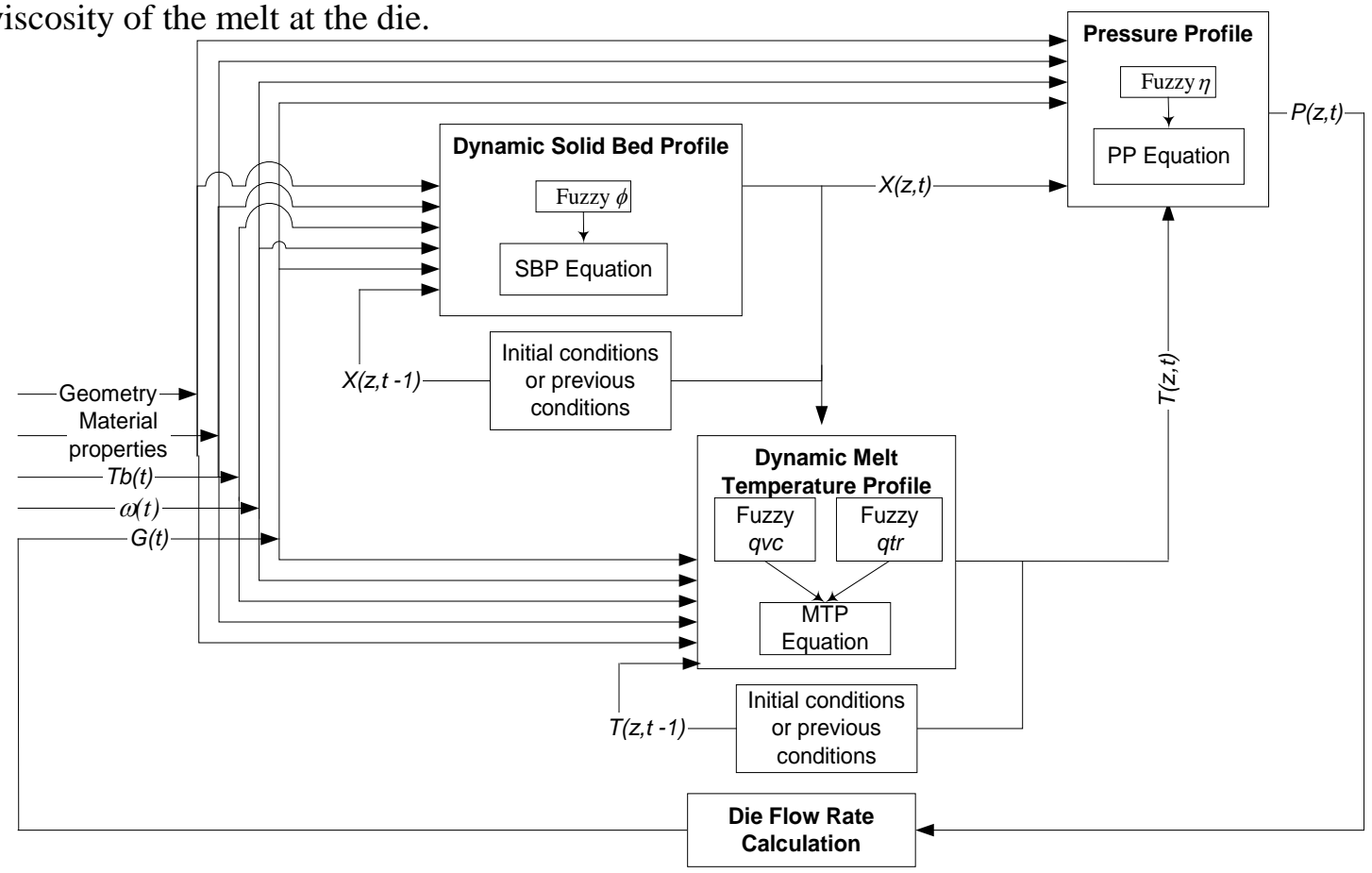

Figure 6: Diagram of model computation 


\section{The Model Evaluation}

Evaluation of the developed model is performed by first checking the consistency of the model prediction to the theoretical analysis. The transient responses of the extrusion are predicted when step changes in the screw speed and barrel temperature are applied separately. Figures 7a, 7b and 7c show the responses of the temperature, pressure and flow rate at the die when a step change is applied to the screw speed. The screw initially rotates at a speed of 60 revolutions/minute (rpm) and the extrusion process is assumed to be operating under a steady-state condition. The dashed lines represent the steadystate responses of the process; the dotted lines represent the responses of the system to a positive step change from $60 \mathrm{rpm}$ to $70 \mathrm{rpm}$, whereas the solid lines are the responses to a negative step change from $60 \mathrm{rpm}$ to $50 \mathrm{rpm}$. In Figure 7a, the model predicts a slow temperature increment when a positive screw speed change is applied. The increase in the temperature of the melt is chiefly attributed to the heat generated from the viscous heat dissipation, which increases with the screw speed and vice versa. Figures $7 \mathrm{~b}$ and 7c show the model predicts immediate changes in both pressure and flow rate at the die; an overshoot can be seen before decaying to a new steady-state value. The responses of the pressure and flow rate at the die are dependent on one another, which is given in Equation 13.

The model is also evaluated for a step change to the barrel temperature setting; assuming that a step change in the barrel temperature can be practically applied. The responses of the melt temperature, pressure and flow rate at the die are shown in Figures 8a, 8b and 8c. The three investigated cases are indicated in the legends. Figure 8a shows that the model predicts an increase in the melt temperature when the barrel temperature is set higher. The higher barrel temperature results in more heat energy being transferred through the barrel wall to melt the solid polymer. Consequently, the melting mechanism commences earlier and the solid is completely melted sooner than the case where lower barrel temperature is applied. The melt temperature shows an inverse response at the early stage when a step change of $180{ }^{\circ} \mathrm{C}$ to $190{ }^{\circ} \mathrm{C}$ is applied to the barrel temperature. Logically, the inverse response should not appear when the barrel temperature is increased. This error is mainly contributed by the simplifying assumptions of the semi-physical model. It is obvious especially when the step change of the barrel temperature is small. 
Figure 8b and 8c show that the melt pressure and flow rate will increase when the barrel temperature is set higher. The increments are attributed to the excess energy supply upon the completion of the melting process. However, the changes in the pressure and flow rate at the die are relatively small when compared with those due to the step changes in the screw speed as shown in Figures 7b and 7c.

a)

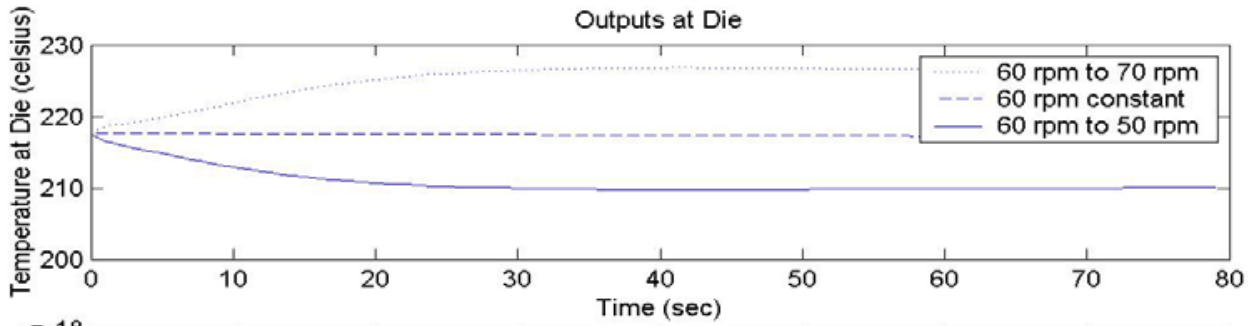

b)

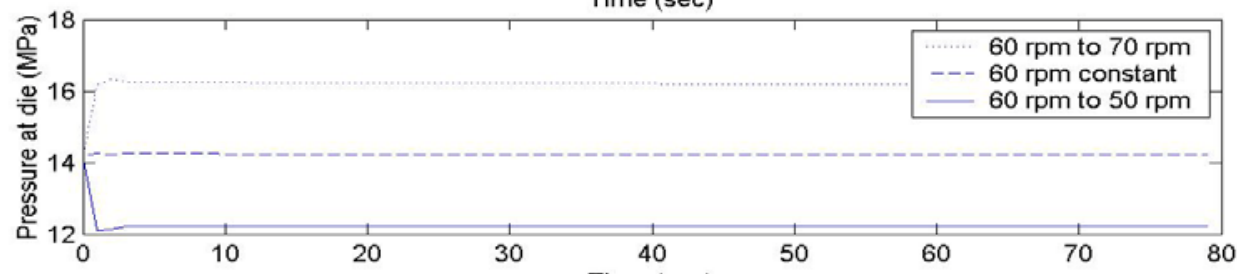

c)

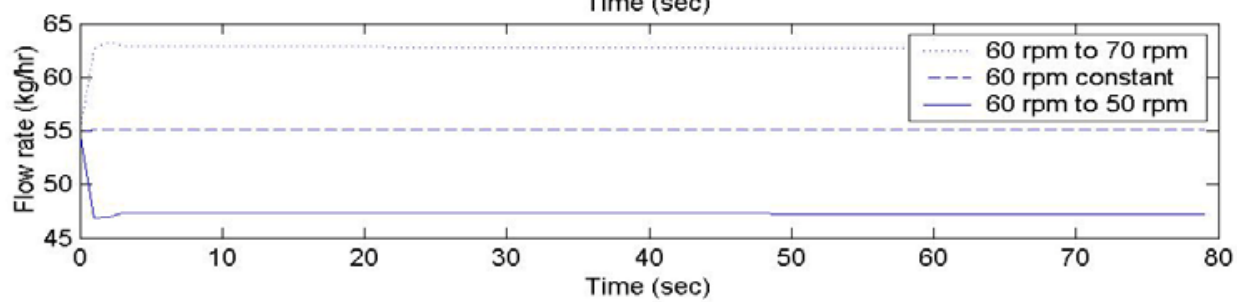

Figure 7: Transients responses of outputs at the die for step changes in screw speed.

a) temperature, b) pressure, and c) flow rate.
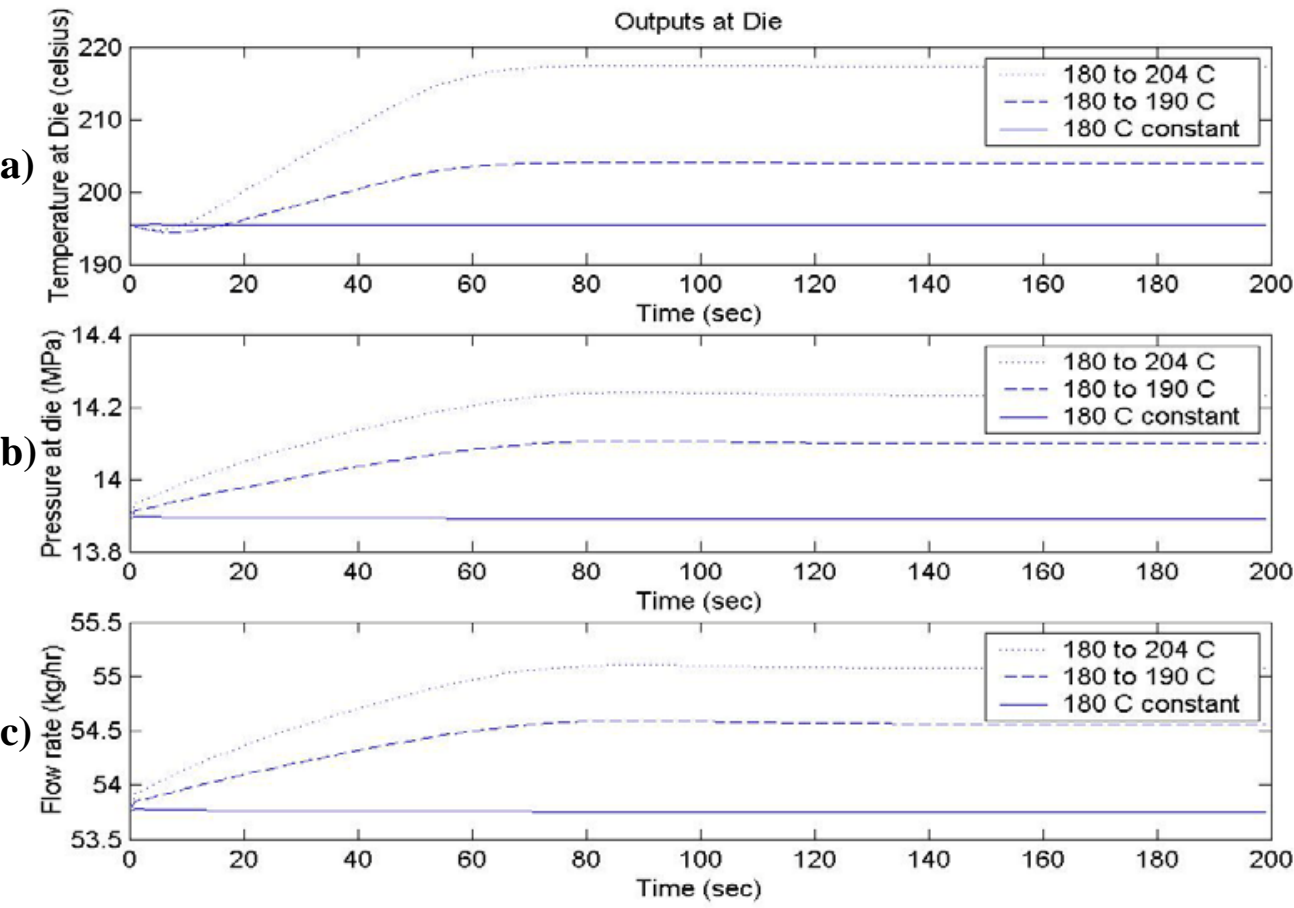

Figure 8: Transients responses of outputs at the die for step changes in barrel temperature.

a) temperature, b) pressure, and c) flow rate. 
The evaluations presented earlier are with the assumption that the model truly describes the behaviour of the extrusion. The assumption might invalid as the actual operating conditions are subjected to the disturbances. Figure 9 shows the results of evaluating the effectiveness of model adaptive capability when the properties of input material vary during the operation. The evaluation is performed to a limit that different input material is being processed. The star-dotted points represent the published experimental measurements of the melt temperatures at five locations distributed along the barrel, when the new material (High Density Polyethylene) is being processed. The dotted line shows the initial prediction of the semi-physical model. The prediction error is obvious as the approximations of the submodels are based on the information of old material (Low Density Polyethylene) before adaptation. The smooth line shows a reasonable good prediction after the adaptation. The sub-models have been tuned automatically to approximate the operational-sensitive parameters in the new operating environment.

The model is further evaluated when it is adopted as the predictive model in the control scheme namely the Fuzzy Supervisory Indirect Learning Predictive Controller (FsiLPC) [12]. Figure 10 indicates that without the model adaptation, both of the plant outputs at die diverge if the modelling error exists. When the model adaptation commences, specifically when the approximations of the sub-models have been tuned automatically according to the varying operating conditions, the melt temperature and the pressure are regulated within an acceptable tolerance as shown in Figures 11a and 11b respectively.

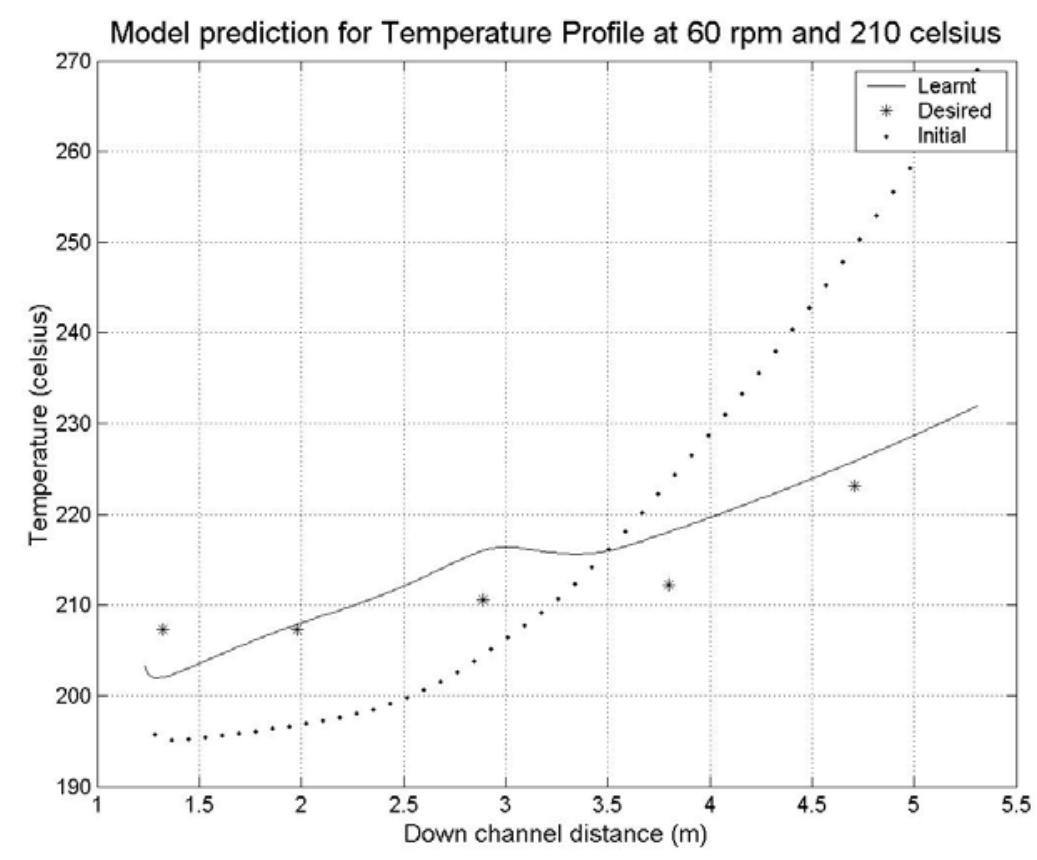

Figure 9: The effectiveness of model adaptive capability for different processing material 

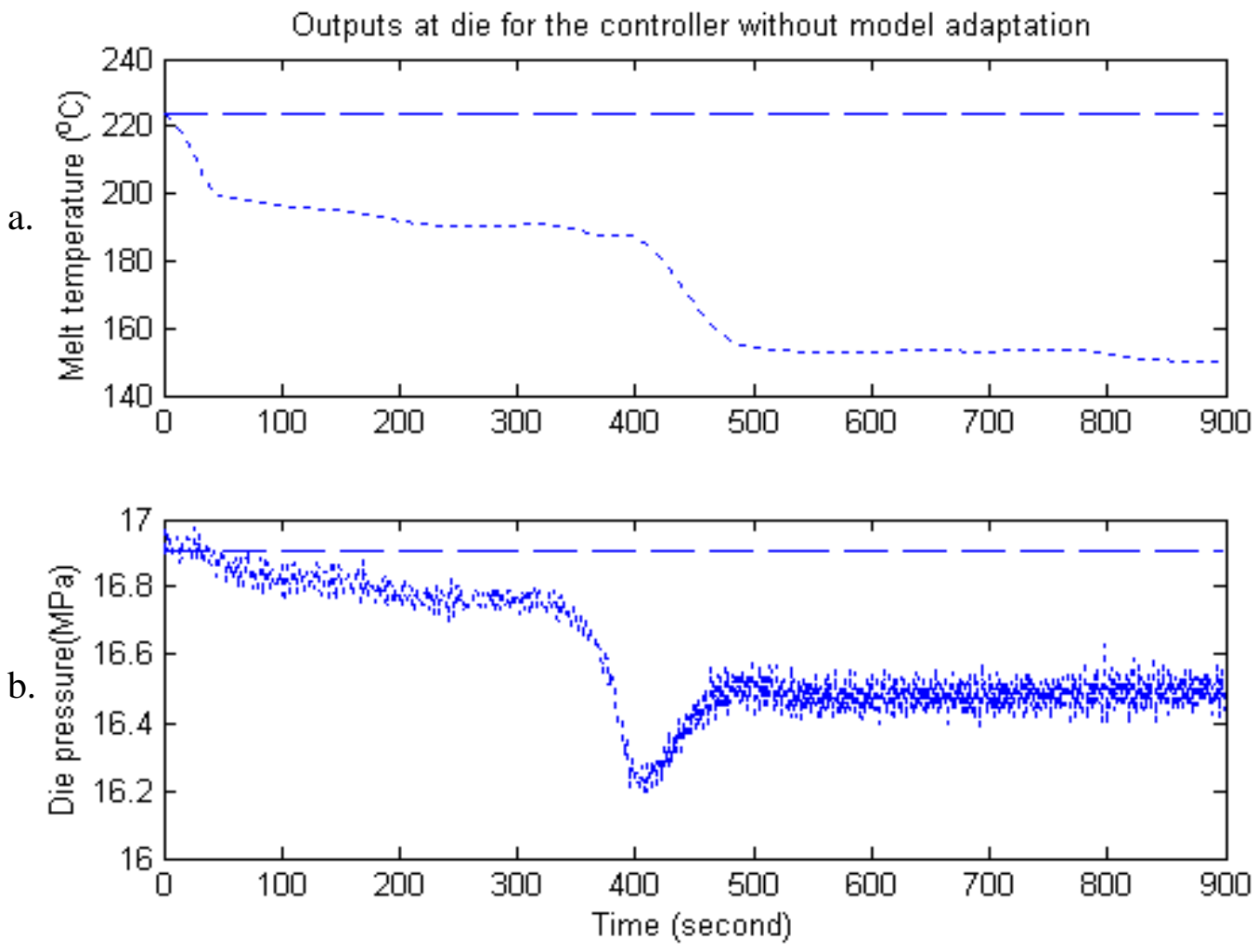

Figure 10: Extrusion control with modeling error without model adaptation

a) melt temperature at die b) melt pressure at die
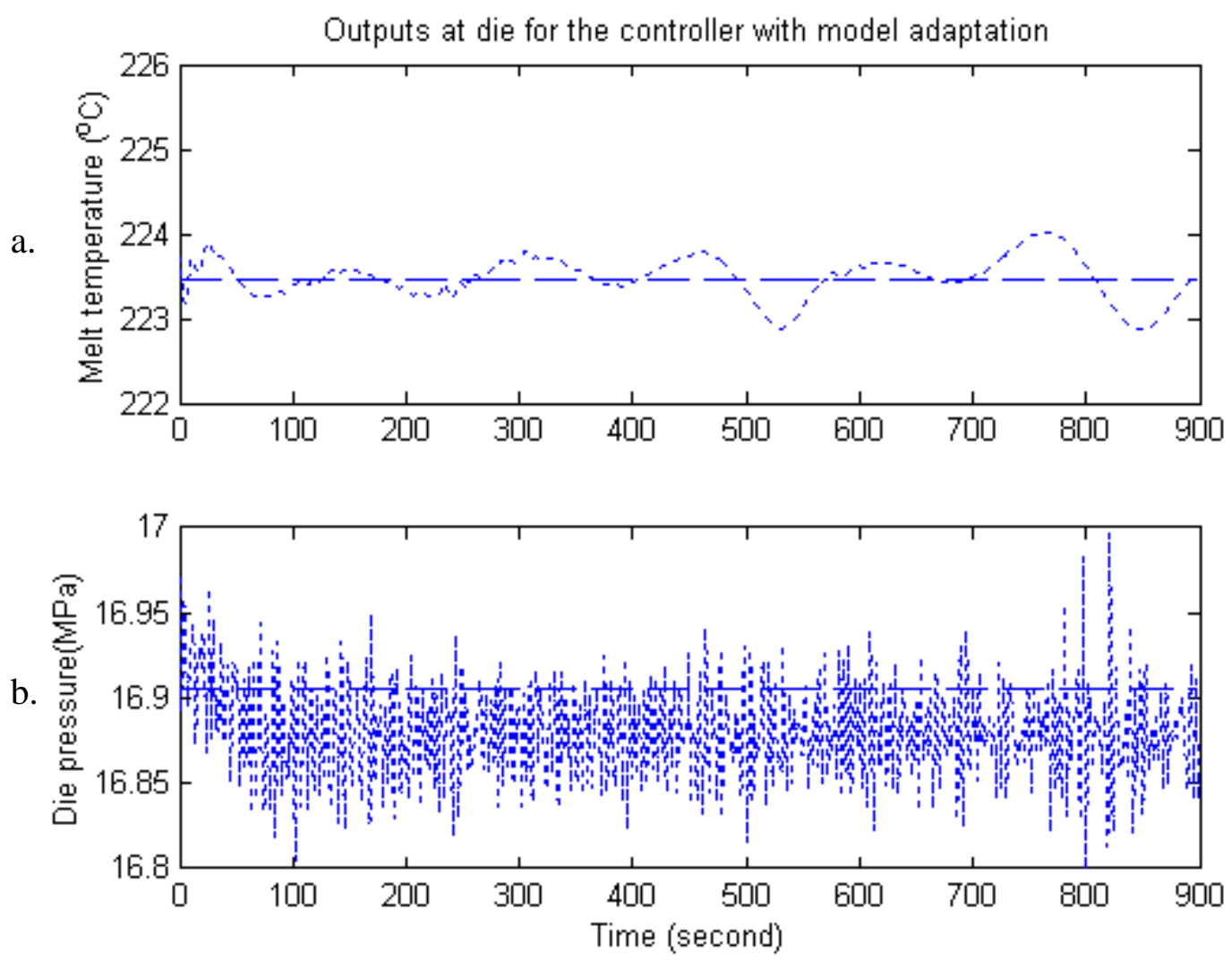

Figure 11: Extrusion control with modeling error with model adaptation

a) melt temperature at die b) melt pressure at die 


\section{Conclusions}

The paper highlighted some of the problems associated with the polymer extrusion modelling for realtime control applications. A semi-physical dynamic model has been developed to estimate the melt temperature, pressure and flow rate at the die. These parameters are regarded as the indicators of performance of the extrusion operation. Fuzzy rule-based sub-models were applied as a means to capture the non-linearity characteristics of the operational-sensitive parameters. Global optimal structures for the sub-models were established by a GA-Fuzzy algorithm. The gradient-based error back-propagation algorithm was implemented only to stimulate the parameters convergence by 'climbing the remaining hill' and to equip the models with an adaptive ability. The optimised structure with smaller number of membership functions and rules would help to increase the interpretability while avoiding the problem of over-parameters. Besides, the speed of simulation would also improve as the sub-models contain fewer parameters.

The model was first evaluated in the basis of consistency to the theoretical analysis. The model predictions in general adhered to the theory when extrusion process was simulated with step changes in the manipulating parameters. Further evaluations were performed to check the effectiveness of submodels adaptations. The results showed that the sub-models, which represent the operational-sensitive parameters had effectively adapted to the changing environment.

Future work is planned to fully evaluate the conditions for adopting the semi-physical model in the design of Fuzzy Supervisory Indirect Learning Predictive Controller in the extrusion control. The advantage of the methodology lies on its multivariable supervisory ability. The effects on the output parameters are considered before a control action such as altering the screw speed is implemented. With this ability, an optimum control function can be ascertained. 


\section{References}

[1] C. Rauwendaal, Polymer Extrusion (Hanser Publishers, New York, 1986).

[2] M.H. Costin, P.A. Taylor and J.D. Wright, A critical review of dynamic modelling and control of plasticating extruders, Polymer Engineering And Science 22(7) (1982) 393-401.

[3] A.K. Kochhar and J. Parnaby, Dynamical modelling and control of plastics extrusion processes, Automica 13 (1977) 177-183.

[4] B. Yang and L.J Lee, Process control of polymer extrusion. Part I: Feedback control, Polymer Engineering and Science 26(3) (1986) 197-204.

[5] S.H. Chiu and S.H. Pong, In-line viscosity control in an extrusion process with a fuzzy gain scheduled PID controller, Journal of Applied Polymer Science 74 (1999) 541-555.

[6] H.A.B. Braake, J.A. Roubos and R. Babuska, Semi-mechanistic modeling and its application to biochemical processes, Fuzzy Logic Control: Advances in Applications (1999) 205-226.

[7] Z. Tadmor, S.D. Lipshitz and R. Lavie, Dynamic model of a plasticating extruder, Polymer Engineering and Science, 14(2) (1974) 112-119.

[8] L.P. Tan, A. Lotfi and E. Lai, Hybrid Soft Computing Methods in Semi-physical Polymer Extrusion Model Identification, $2^{\text {nd }}$ European Symposium on Intelligent Technologies (2002) 652-656.

[9] N. Brauner, R. Lavie, Z. Tadmor, Control of plasticating extruder. 3rd IFAC Conference on Instrumentation and Automation in the Paper, Rubber and Plastic Industry (1977) 353-360.

[10] O. Cordon, F. Herrera, F. Hoffmann and L. Magdalena, Genetic Fuzzy Systems: Evolutionary Tuning and Learning of Fuzzy Knowledge Base (World Scientific Publishing, Singapore, 2001).

[11] H. Surmann and A. Selenschtschikow, Automatic generation of fuzzy logic rule bases: Examples I, Proceedings of NF '2002 (2002) 75-81.

[12] L.P. Tan, A. Lotfi, E. Lai and J.B. Hull, Development and Application of a Fuzzy Supervisory Indirect Learning Control, $3^{\text {rd }}$ European Symposium on Intelligent Technologies (2003), 346-352. 